\title{
Grip Strength and North American Collegiate Football Performance Indicators
}

\author{
Ryan Otterson, $\odot$ Mark DeBeliso
}

Southern Utah University, Department of Kinesiology and Outdoor Recreation, Cedar City, UT, USA

\begin{abstract}
Football athletes are often tested in their ability to display high levels of strength and power to determine their sporting prowess. One indicator of total body strength in the general population is hand grip strength (HGS) which has also been used to stratify level of success in a variety of sports. It is of interest to determine what relationship HGS has with other indicators of football performance. This study examined the correlation between HGS as it relates to indicators of football sport performance: vertical jump (VJ), 18.3 meter sprint (13.8MS), and one repetition maximum back squat (1-RM BSQ) among North American collegiate football players. Sixteen $(n=16)$ male participants from a NCAA division II football program (age: $21.3 \pm 1.7$ years, height: $183.6 \pm 9.1 \mathrm{~cm}$, mass: $99.6 \pm 17.7$ $\mathrm{kg}$ ) were assessed for HGS, VJ, 18.3MS, and 1-RM BSQ. Athletes performed a dynamic warm-up followed by two trials of each assessment: HGS, VJ, and 18.3MS. A recent 1-RM BSQ as recorded by the college's strength and conditioning staff was used as the measure of BSQ strength. Pearson correlation coefficients (r) were determined for HGS and football performance tests as well as HGS to body mass ratio and football performance tests. Moderate correlations were found between HGS and VJ, as well as HGS and 18.3MS (r=0.41 , and $\mathrm{r}=0.49$; respectively). HGS to body mass (HGS/BM) ratio also demonstrated moderate correlations with VJ ( $\mathrm{r}=0.50)$ and 18.3MS times $(\mathrm{r}=-0.41)$. No significant correlation was found between HGS and 1-RM BSQ ( $\mathrm{r}=0.09$ ) or HGS/BM with 1-RM BSQ/BM ( $\mathrm{r}=-$ 0.13). Within the parameters of this study, HGS as it relates to an athlete's body mass has a moderate relationship with performance in the VJ and the 13.8MS.
\end{abstract}

Keywords. Collegiate, dynamometer, football.

\section{Introduction}

Hand grip strength (HGS) is the amount of force the forearm and hand musculature can produce in a given position and is commonly assessed using hand grip dynamometry (Roberts et al., 2011). In HGS, typically the maximal voluntary isometric force of the forearm flexors are assessed with a hand held device designed to measure the maximal gripping force one can produce. Measures of HGS have high utility in assessing health and sport performance indicators. It is an easy to perform test with high reliability and low fatigue, making it a very practical field test in numerous strength and conditioning, epidemiological, and clinical settings (Cronin et al., 2017; Roberts et al., 2011).

In multiple studies, HGS has been shown to correlate with greater overall body strength and longevity, and is associated with greater functionality as one ages. Rantanen et al. (1999) indicated that HGS at middle age was indicative of strength and activity levels in old age through their 25-year prospective cohort study of JapaneseAmericans. Similarly, DeBeliso et al. (2015a, 2015b) demonstrated strong positive correlations between HGS and measures of lower and upper body strength and functionality in mature adults. HGS was found to be predictive of long-term survival among those who lived past the age of 85 in the cohort study by Granic et al. (2017) of 845 men and women from Northern England. Granic et al. (2017) also stated that HGS was reflective of total body muscle strength and predictive of mortality risk in the elderly. Some research has even shown rates of decline in HGS to moderately coincide with rates of decline in measures of cognitive decline in the elderly (Praetorius et al., 2016). In younger demographics, HGS has also been shown to increase with increasing height and weight among girls and boys between the ages of 8 and 20 years 
old (Wind et al., 2010). Wind et al. (2010) concluded that increases in total muscle mass account for increases in HGS as shown by normal growth in children and young adults.

HGS appears reflective of muscle strength and physical capabilities, especially in ageing populations, but do physical activity interventions aimed at increasing HGS also increase overall strength? Conversely, do training interventions aimed at increasing overall strength improve HGS? Two studies on physical activity intervention's effects on HGS suggested mixed results. Dash \& Telles (2001) found that a yoga training intervention significantly improved HGS in children (12-15 years old), adults (23-50 years old), and adults (22-43 years old) with rheumatoid arthritis. However, research by Santanasto et al. (2017) found no significant change in HGS in older adults following a lower body strength and balance training intervention that resulted in increases in chair stand strength and $400 \mathrm{~m}$ walking speed. These mixed results indicate that HGS may be an indicator of health, functionality, and overall body strength in non-athletic populations, but does not precisely coincide with these measures.

As HGS is an indicator of overall strength, it follows that it has also been shown to be a predictor of sporting ability in a variety of different sports. A recent meta-analysis of the role of HGS in sport performance examined HGS's role in predicting sport performance, and its' correlation to performance indicators in combat sports, ball sports, field sports, and strength sports (Cronin et al., 2017). HGS was found to be predictive of sporting ability in combat and strength sports, but not in ball or field sports. HGS correlated strongly with rankings in professional boxers (Guidetti et al., 2002), and was also among the strongest predictors of successful vs. unsuccessful GrecoRoman wrestlers at the national level (Nikooie et al., 2017). Nikooie et al. (2017) also found that HGS per kilogram of body mass was higher in successful wrestlers and in those who had more wrestling experience, indicating that stronger wrestlers seemed to win more often than their weaker counter parts. HGS differs with the type of sport athletes compete in as evidenced by findings from
Iermekov et al. (2006) that HGS was significantly greater in martial artists involved in grappling sports (wrestling, judo, sambo) than in martial artists involved in striking sports (karate, taekwondo, hand-to-hand combat). This idea is further supported by HGS's strong predictive abilities in strength sports contrasted with its' lack of predictive power in ball and field sports. Fry et al. (2006) found that HGS was one of five field tests that could differentiate elite junior weightlifters from elite senior weightlifters, and Schoffstall et al. (2010) found HGS to correlate strongly with raw powerlifting totals in a state championship meet. Grapplers and strength athletes both use grip heavily in competition and in the training involved for their respective sports so it comes as no surprise that HGS is indicative of ability in these sports.

Studies of HGS's relationship to athletic performance in ball and field sports have yielded mixed results (Cronin et al., 2017). James et al. (2016) found that HGS correlates moderately with measures of soccer performance such as T-test time, vertical jump, and sprint time in adolescent boys aged 11-17 years. James et al. (2016) ultimately concluded that in younger athletes, age is the strongest predictor of scores on all these tests, HGS included. This notion may suggest that HGS correlates well with overall strength and performance, but that maturity and the associated strength increases are more important in younger athletes as opposed to mature athletes. In North American Football, HGS was found to be higher in older, more experienced athletes (professional compared to collegiate), but it was not meaningfully correlated with rankings among NFL players (Straub, 1979). Shields et al. (1984) found no significant differences in HGS between NFL veterans and non-starters and that HGS was not a predictor of ability.

As North American football is a sport that requires high levels of total body strength and power, and more of an emphasis is placed on these qualities as the sport of football continues to evolve, it is possible that HGS plays a bigger role in talent identification than previous studies have alluded to, especially in young maturing populations. It appears that few studies have 
looked at the association between football performance and HGS overall (Ağbuğa et al., 2009; Shields et al., 1984; Straub, 1979) and that there is a paucity of research regarding modern day football players and HGS. As such, it is possible that HGS may be useful as a practical field test in order to quantify overall body strength and to identify talent. Furthermore, HGS as it relates to body mass (HGS/BM) may be an even better indicator of sporting performance than HGS alone.

North American football talent identification often uses measures of lower body strength and power such as the vertical jump (VJ), the long jump, and short sprints (40 yds or 36.6MS) to assess a player's physical abilities. While some have criticized these tests as lacking the ability to predict performance during an NFL (National Football League) career, these tests are still widely used to determine ability and are often trained for during an athlete's college career (Lyons, Hoffman, Michel, \& Williams, 2011). With that said, this study attempted to determine if there was any meaningful relationship between: measures of grip strength HGS, HGS/BM, and one repetition maximum back squat (1-RM BSQ), 1-RM BSQ per kilogram of body mass (1-RM BSQ/BM), a short sprint time (20 yds or $18.3 \mathrm{MS}$ ), and VJ. It was hypothesized that HGS measures will be meaningfully associated with physical performance measures important to North American football performance.

\section{Methods}

\section{Participants}

A convenience sample of male NCAA Division II football players were recruited from Dixie State University (DSU) in St. George, Utah. All participants were healthy men playing in various positions on the DSU football team. Participants were free of any neuromuscular, orthopedic, or neurological conditions that might interfere with physical activity. They were recruited via flyers distributed with the DSU Strength and Conditioning staff.
Prior to any testing or assessment, permission from the Institutional Review Board was obtained. Each participant was provided with a written informed consent form to read and sign before they were included in the study. It was made clear to all participants that participation was strictly voluntary and could be withdrawn at any time for any reason, and that no compensation would be provided.

\section{Instruments and Apparatus}

Participants took part in a performance testing session measuring their HGS, 18.3MS sprint time, and VJ; measurements of height and weight were also taken. Hand grip strength was measured using a Saehan hand grip dynamometer (SH5001; Saehan, Yangdeok-Dong, South Korea) (Reis \& Arantes, 2011), borrowed from Dixie Regional Medical Center's hand therapy department.

The sprints (18.3MS) were timed with a Brower Timing System (2014 model, Draper, Utah) loaned from Dixie Regional Medical Center's (St. George, Utah, US) Sports Performance Department. Vertical jumps were recorded with a Vertec device located in the DSU Strength and Conditioning room. Height was measured with a wall mounted stadiometer while weight was measured on an electronic scale; both devices were located in the DSU weight room.

\section{Procedures}

Testing was performed in one session lasting about 1.5 hours in the Dixie State University weight room and adjacent football field. All testing was conducted as part of a normal strength and conditioning training session for the football team. Upon arrival, the subjects performed a dynamic warm-up protocol as set forth by the Strength and Conditioning coach. The warm-up consisted of the following exercises performed over an 8.3 meter distance: Toe walks, heel walks, knee pulls to the chest, ankle grabs, quad stretch with single-leg Romanian deadlift, soldier march, toe pulls, inchworms, lateral lunges, carioca, and two sets of a light sprints.

Following the dynamic warm-up, participants' height, weight, name, and age were recorded along 
with their right or left handedness. Height and weight were both measured without shoes, but weight was measured with whatever clothes participants were wearing to train in. Following anthropometric data collection, participants were split into two separate groups to perform either the VJ or 18.3MS. Those who performed the 18.3MS first then performed the VJ and vice-versa. This was done to help speed up the testing process where testing order was not thought to influence results. There was a 3-5 minute rest separating the VJ and 18.3MS trials.

The 18.3MS sprints were laser timed on the DSU football field adjacent to the weight room. Participants lined up on the goal line at one end of the field and were instructed to run through the Brower laser gate on the command of "go" by the tester. Each participant was told to set up in a twopoint stance, with their preferred foot forward. They could lean forward as much as desired so as their toes did not cross the goal line and they were able to remain motionless until the "go" command. Brower TC-PhotoGates were set up at a distance of 18.3 meters from the start line at around knee height and with 2.7 meters distance between them for the participants to run through. Each sprint was manually started using the Brower TC Timer on the command of "go" by the tester. Participants performed two trials with 3-5 minutes rest between trials. Short sprints have a high test-retest reliability $(r \geq 0.95)$ when fatigue is not a factor (Miller, 2012).

All participants performed two trials of the VJ test after measuring each participants' reach with the Vertec and allowing them a warm-up jump to familiarize themselves with the testing protocol and device. Reach was measured by having each participant stand next to the Vertec with their dominant arm raised and extended maximally while keeping their feet flat on the ground. Athletes were instructed to jump as high as possible without taking a lead in step and to reach for the highest vane they could touch on the Vertec device using the hand they measured reach with. Athletes were instructed to use the counter jump movement which requires the swinging of the arms. Markovic et al. (2004) determined an inter- trial reliability of $\mathrm{r}=0.90$ for the countermovement VJ jump with a reach.

After performing jump and sprint trials, HGS was assessed using the Saehan dynamometer. Participants were instructed to sit with their shoulder adducted and neutrally rotated (at their side), with their elbow bent to $90^{\circ}$ of flexion and to maintain a neutral forearm (Fess, 1981). Participants received instructions to squeeze the dynamometer as hard as possible for a duration of 3 seconds. Subjects performed two trials with their dominant hand, resting 1 minute between trials. The Saehan dynamometer was shown to have high test-retest reliability ( $\mathrm{r}=0.981$ right hand; $\mathrm{r}=0.985$ left hand) when testing was performed similar to as described above (Reis \& Arantes, 2011).

Each athlete's most recent 1-RM BSQ score was obtained from the DSU Strength and Conditioning Coach. The athletes recently had their 1-RM BSQ assessed as part of their formal strength and conditioning program (within the prior 2-3 months). This was done at the request of the coaching staff so as not to put the football players at increased risk of injury and to avoid significantly disrupting their training schedule right before the start of the football season. The 1-RM BSQ assessment has been shown to have test-retest reliability ranging from between $\mathrm{r}=0.92-0.99$ (McBride et al., 2002; Sanborn et al., 2000).

\section{Design and Analysis}

The variables assessed/collected in this study were: HGS (kgs), body mass (kgs), 18.3MS time (seconds), 1-RM BSQ (kgs), and VJ (cm). Further, the 1-RM BSQ and HGS were normalized to body mass for statistical analysis. Associations between HGS the VJ, 1-RM BSQ, and 18.3MS time were calculated with Pearson correlation coefficients (PCC or r). Likewise, the relationships between HGS/BM and measures of the VJ, 1-RM BSQ/BM, and $18.3 \mathrm{MS}$ time were calculated with Pearson correlation coefficients (r). Participants' HGS scores were also compared to normative data for American men (Wang et al., 2018). Significance for the study was set a priori at $\alpha \leq 0.05$. Statistical analyses and data management were completed in an MS Excel 2013. The Excel spreadsheet was peer 
reviewed as advanced by AlTarawneh \& Thorne (2017).

\section{Results}

Sixteen male NCAA Collegiate North American football players volunteered for and completed the study without incident. The participant demographics and anthropometric measurements are listed in Table 1. Average scores of the performance tests are found in Table 2. The participant's HGS is compared with normative data in Table 3. The PCC's between HGS the VJ, 1RM BSQ, and 18.3MS are presented in Table 4. Likewise, the PCC's between HGS/BM the VJ, 1RM BSQ/BM, and 18.3MS are provided in Table 5.

Table 1

Participant descriptive data (Mean \pm SD).

\begin{tabular}{llll}
\hline & Age (years) & Height $(\mathrm{cm})$ & Mass $(\mathrm{kg})$ \\
\hline Male $(\mathrm{n}=16)$ & $21.3 \pm 1.7$ & $183.6 \pm 9.1$ & $99.6 \pm 17.7$ \\
\hline
\end{tabular}

Normative reference percentiles were obtained from Table 1 of Wang et al. (2018)'s study using the Jamar dynamometer and are based on hand dominance, gender, and age. Measures from the Saehan dynamometer used in the current study are interchangeable with the Jamar device (Reis \& Arantes, 2011).

\section{Discussion}

The purpose of this study was to determine if there was a meaningful relationship between HGS and physical characteristics considered indicative of North American football performance. The research hypothesis was that HGS, and an athlete's
HGS/BM ratio, would meaningfully correlate with performance in the 18.3MS, VJ, 1-RM BSQ, and 1RM BSQ/BM. The results of the study were mixed in regards to the research hypothesis. While moderate correlations were found between absolute HGS and VJ and 18.3MS performance, they were in the direction opposite the hypothesis. The results of this study corroborated those of other studies that showed higher values of HGS in individuals with greater BM (Cronin et al., 2017; Shields et al., 1984), which may account for the moderately negative correlation of HGS with VJ height and the moderate relationship of higher HGS with slower 18.3MS times. However, when looking at HGS in relation to each athlete's BM, results supported the tenants of the research hypothesis. Specifically, athletes with higher ratios of HGS/BM demonstrated moderate correlations with faster 18.3MS times and a higher VJ.

All but three subjects in this study scored in the upper $90^{\text {th }}$ percentile for HGS among American men. The three who did not score in the upper $90^{\text {th }}$ percentile were still above the average with two subjects scoring between the $50^{\text {th }}$ and $75^{\text {th }}$ percentile and one subject scoring between the $75^{\text {th }}$ and $90^{\text {th }}$ percentile for HGS (Wang et al., 2018). The current sample of Division II football players had an average 1-RM BSQ of $182.0 \pm 35.6 \mathrm{~kg}$ which would place them between the $60^{\text {th }}$ and $70^{\text {th }}$ percentile rank amongst NCAA Division III players and between the $50^{\text {th }}$ and $60^{\text {th }}$ percentile rank amongst NCAA Division I players according to strength norms found in Essentials of Strength Training and Conditioning (2008). This study's average VJ performance $(67.7 \pm 10.3 \mathrm{~cm})$ was just under the average of $69.3 \pm 8.5 \mathrm{~cm}$ found among NCAA Division II football players (Fry \& Kraemer, 1991).

Table 2

Scores of performance variables (Mean $\pm \mathrm{SD}$ ).

\begin{tabular}{cccccc}
\hline HGS $(\mathrm{kg})$ & HGS/BM & VJ $(\mathrm{cm})$ & $18.3 \mathrm{MS}(\mathrm{sec})$ & 1-RM BSQ $(\mathrm{kg})$ & $1-\mathrm{RM}$ BSQ/BM \\
\hline $64.9 \pm 9.5$ & $0.7 \pm 0.1$ & $67.7 \pm 10.3$ & $3.14 \pm 0.27$ & $182.0 \pm 35.6$ & $1.8 \pm 0.3$ \\
\hline
\end{tabular}




\section{Table 3}

Measured HGS compared to reference values.

\begin{tabular}{ccccc}
\hline Participant & Age (year) & HGS $(\mathrm{kg})$ & $\begin{array}{c}\text { Normative Percentile Range } \\
\text { (Actual) }\end{array}$ & HGS/BM \\
\hline 1 & 22 & 64 & $>90$ & 0.74 \\
2 & 19 & 55 & $75-<90$ & 0.59 \\
3 & 23 & 75 & $>90$ & 0.56 \\
4 & 22 & 60 & $>90$ & 0.68 \\
5 & 23 & 50 & $50-<75$ & 0.63 \\
6 & 21 & 66 & $>90$ & 0.62 \\
7 & 20 & 48 & $50-<75$ & 0.55 \\
8 & 21 & 61 & $>90$ & 0.62 \\
9 & 19 & 64 & $>90$ & 0.67 \\
10 & 23 & 60 & $>90$ & 0.49 \\
11 & 20 & 78 & $>90$ & 0.75 \\
12 & 20 & 62 & $>90$ & 0.73 \\
13 & 21 & 76 & $>90$ & 0.57 \\
14 & 25 & 67 & $>90$ & 0.87 \\
15 & 22 & 70 & $>90$ & 0.67 \\
16 & 19 & 80 & $>90$ & 0.84 \\
\hline
\end{tabular}

Table 4

Correlation between performance variables and mean HGS.

\begin{tabular}{cccc}
\hline \multirow{2}{*}{ Performance Variable } & HGS & Significant? & Size \\
\hline VJ & $r$ & $\mathrm{P}<0.05$ & Moderate \\
18.3MS & -0.41 & Yes & Moderate \\
1-RM BSQ & 0.49 & Yes & \\
\hline
\end{tabular}

\section{Table 5}

Correlation between performance variables and HGS/BM.

\begin{tabular}{cccc}
\hline \multirow{2}{*}{ Performance Variable } & HGS/BM & Significant? & Size \\
\hline VJ & $r$ & P $<0.05$ & Moderate \\
18.3MS & 0.50 & Yes & Moderate \\
1-RM BSQ/BM & -0.41 & Yes & \\
\hline
\end{tabular}

Results from this study show that higher levels of HGS/BM correlate moderately with a greater ability to jump higher and sprint faster which is in agreement with Cronin et al.'s (2017) statement that HGS seems to be a covariate with impulsive ability (i.e., sprinting \& jumping) among elite athletes. This study did not measure whether these traits relate to position, whether the players were starters or not, or if these attributes are indicative of being a higher-ranking football player or team. 
Hand grip strength did not correlate with 1-RM BSQ nor did HGS/BM correlate with 1-RM BSQ/BM. Back squats, even though primarily a lower body exercise, are also thought to be a good indicator of total body strength, but there was no meaningful (or significant) relationships found between the measures of the 1-RM BSQ and HGS. This is somewhat surprising as muscular strength is strongly associated with athletic performance in sports skills such as running, jumping, and changeof-direction ability (Suchomel et al., 2016). It is possible that the homogeneity of a sample of highlevel athletes could account for the lack of a meaningful correlation between metrics of the 1RM BSQ and HGS. However, the supposition of homogeneity seems at odds with the moderate correlations found between HGS/BM and VJ as well as 18.3MS times in this study's sample.

The current study is among a few comparing metrics of HGS with common North American football performance measures. The results of the current study were contrary to those previously reported (Ağbuğa et al., 2009; Cronin et al., 2017; Shields et al., 1984; Straub, 1979). In a review by Cronin et al. (2017) only a few studies of North American football player performance variables and HGS measures were referenced. Cronin et al. (2017) suggested that HGS was important to North American football performance for the purpose of throwing, but provided no direct evidence to support this supposition. Ağbuğa et al. (2009) compared HGS/BM and 1-RM bench press/BM scores among collegiate North American football players and concluded that HGS/BM could not be used to predict the 1-RM bench press/BM. Shields et al. (1984) examined HGS, body weight, leg strength, VO2 max, and various other measures of North American professional football players with respect to experience and position played. Shields et al. (1984) found non-significant differences in HGS between veterans and non-starters. Straub (1979) exclusively examined HGS to determine the association between HGS and football playing performance (by rankings) among college and professional North American football players. Straub (1979) concluded "that grip strength was not correlated meaningfully with football-playing performance". However, the results of the current study cannot be directly compared to Straub's study which assessed HGS with a tensiometer device (Model T5, Pacific Scientific Co., Anaheim, California).

Football athletes at the collegiate level are expectedly strong when compared to the general population. North American collegiate and professional players could be considered as a type of strength athlete given the amount of resistance training players have been exposed to. Prior research regarding HGS and strength athletes (power lifters and Olympic lifters) suggests that HGS is associated with performance (Fry et al., 2006; Kozlik \& Boswell, 2010). The aforementioned relationship between HGS and strength athlete performance would intuitively suggest a relationship between HGS and lower body strength, which was not supported by the results of the current study.

Limitations to this study were the small sample size and the reliance on past scores of 1-RM BSQ scores. It is possible that BSQ form and depth was not standardized among the athletes who were tested. However, these 1-RM BSQ scores were collected by the same strength and conditioning professional. The use of recently collected 1-RM BSQ scores could also imply that the participant's current lower body strength was not precisely represented by data analyzed in the current study. This could account for the lack of correlation between HGS and the 1-RM BSQ, whereas 18.3MS speed and VJ demonstrated moderate correlations with HGS. With that said, correlations are based on the rank order of a pair of scores, in this case, the participant's 1-RM BSQ rankings compared with the rankings of HGS. So, while the 1-RM BSQ scores might not have been a precise reflection of the participant's lower body strength, it is reasonable to believe the 1-RM BSQ scores were reflective of the rank order of the participant's lower body strength. Hence, it is realistic to assume that the time of the 1-RM assessment had little to do with the lack of association between the participant's HGS and the 1-RM BSQ scores 
Future research should be conducted with athletes from a variety of different sports, genders, and levels of ability to determine if meaningful relationships exist between metrics of HGS and measures of athletic ability exist. Other genres of research could be conducted to determine if certain resistance training protocols (longitudinal) and exercise modalities are effective for the purpose of developing HGS and to what extent the newly developed HGS is associated with improvements in total body strength (or athletic tasks). Grip strength is useful in sports that explicitly require the use of the hands to couple with an implement or an opponent such as CrossFit, weightlifting, gymnastics, and combat sports (Fry et al., 2006; Guidetti et al., 2002; Haynes \& DeBeliso, 2019; Nikooie et al., 2017; Ruprai et al., 2016). However, evidence to indicate the importance of HGS in a general athletic sense is still lacking.

In conclusion, collegiate football players demonstrate high levels of HGS, lower body strength, and lower body power. The ratio of the players' HGS/BM moderately correlated with better performance in the VJ and the 18.3MS. Larger athletes tended to demonstrate higher measures of HGS but were also slower in the 18.3MS and jumped lower in the VJ. In this study, athletes who demonstrated higher ratios of HGS/BM performed better in indicators of football performance. Assessing an athlete's HGS/BM requires minimal time, little expertise (administration and athlete), and uses a low-cost hand dynamometer. Coaches and strength and conditioning professionals might consider the assessment of HGS/BM as a useful field test in determining athletic potential.

\section{Conflict of Interest Declaration}

No funding was received for this research. The authors have no conflict of interest related to this research. This research has not been previously published.

\section{References}

Ağbuğa, B., Konukman, F., \& Yilmaz, I. (2009). Prediction of upper body strength by using grip strength test in division II American college football players' grip strength. Hacettepe Journal of Sport Science, 20, 16-23.

AlTarawneh, G., \& Thorne, S. A pilot study exploring spreadsheet risk in scientific research. Retrieved from http://arxiv.org/abs/1703.09785. March 2017.

Baechle, T.R., \& Earle, R.W. (2008). Essentials of strength and conditioning, $3^{r d}$ ed. Champaign, IL: Human Kinetics.

Cronin, J., Lawton, T., Harris, N., Kilding, A., \& McMaster, D.T. (2017). A brief review of handgrip strength and sport performance. Journal of Strength and Conditioning Research, 31(11), 3187-3217. doi:10.1519/JSC.0000000000002149

Dash, M., \& Telles, S. (2001). Improvement in hand grip strength in normal volunteers and rheumatoid arthritis patients following yoga training. Indian Journal of Physiology and Pharmacology, 45(3), 355-360.

DeBeliso, M., Boham, M., Harris, C., Carson, C., Berning, J.M., Sevene, T.G., \& Adams, K.J. (2015a). Grip and body strength measures in the mature adult: A brief report. International Journal of Science and Engineering Investigations, 4(37), 83-86.

DeBeliso, M., Boham, M., Harris, C., Carson, C., Berning, J.M., Sevene, T.G., Adams, K.J., \& Climstein, M. (2015b). Grip strength and functional measures in the mature adult: Brief report II. International Journal of Science and Engineering Investigations, 4(39), 1-4.

Haynes, E., \& DeBeliso, M. (2019). The relationship between CrossFit performance and grip strength. Turk J Kinesiol, 5(1), 15-21.

Fess, E.E., \& Moran, C. Clinical assessment recommendations. Indianapolis: American Society of Hand Therapists Monograph; 1981.

Franchini, E., Takito, M., \& Bertuzzi, R. (2005) Morphological, physiological and technical variables in high-level college judoists. Science of Martial Arts, $1,1-7$.

Fry, A.C., Ciroslan, D., Fry, M.D., LeRoux, C.D., Schilling, B.K., \& Chiu, L.Z. (2006). Anthropometric and performance variables discriminating elite American junior men weightlifters. Journal of Strength and Conditioning Research, 20(4), 861-866.

Fry, A.C., \& Kraemer, W.J. (1991). Physical performance characteristics of American collegiate football players. Journal of Applied Sport Science Research, 5(3), 126-138.

Granic, A., Davies, K., Jagger, C., M Dodds, R., Kirkwood, T. L., \& Sayer, A. A. (2017). Initial level 
and rate of change in grip strength predict all-cause mortality in very old adults. Age and Ageing, 46(6), 970-976. doi:10.1093/ageing/afx087

Guidetti, L., Musulin, A., \& Baldari, C. (2002). Physiological factors in middleweight boxing performance. Journal of Sports Medicine $\mathcal{E}$ Physical Fitness, 42(3), 309-314.

Iermakov, S. S., Podrigalo, L. V., Jagiello, W. (2006). Hand-grip strength as an indicator for predicting the success in martial arts athletes. Retrieved from https://dspace.ukw.edu.pl/handle/item/3911

James, R., Thake, C., \& Birch, S. (2017). Relationships between measures of physical fitness change when age-dependent bias is removed in a group of young male soccer players. Journal of Strength and Conditioning Research, 31(8), 2100-2109.

Lyons, B., Hoffman, B., Michel, J., \& Williams, K. (2011). On the predictive efficiency of past performance and physical ability: The case of the National Football League. Human Performance, 24(2), 158-172.

Markovic, G., Dizdar, D., Jukic, I., \& Cardinale, M. (2004). Reliability and factorial validity of squat and countermovement jump tests. Journal of Strength and Conditioning Research, 18(3), 551-555.

McBride, J.M., Triplett-McBride, T., Davie, A., \& Newton, R.U. (2002). The effect of heavy vs. lightload jump squats on the development of strength, power, and speed. Journal of Strength and Conditioning Research, 16, 75-82.

Meier, J., Quednow, J., \& Sedlak, T. (2015). The effects of high intensity interval-based kettlebells and battle rope training on grip strength and body composition in college-aged adults. International Journal of Exercise Science, 8(2), 124-133.

Miller, T. (2012). NSCA's guide to tests and assessments. Champaign, IL: Human Kinetics.

Miller, T.A., White, E. D., Kinley, K. A., Congleton, J. J., and Clark, M. J. (2002). The effects of training history, player position, and body composition on exercise performance in collegiate football players. Journal of Strength and Conditioning Research, 16:44-49.

Nikooie, R., Cheraghi, M., \& Mohamadipour, F. (2017). Physiological determinants of wrestling success in elite Iranian senior and junior Greco-Roman wrestlers. Journal of Sports Medicine and Physical Fitness, 57, 219-226.
Norman, K., Stobäus, N., Gonzalez, M. C., Schulzke, J., \& Pirlich, M. (2011). Hand grip strength: outcome predictor and marker of nutritional status. Clinical Nutrition, 30(2), 135-142. doi:10.1016/j.clnu.2010.09.010

Praetorius, M., Johansson, B., \& Hassing, L. B. (2016). I forgot when I lost my grip-strong associations between cognition and grip strength in level of performance and change across time in relation to impending death. Neurobiology of Aging, 38, 68-72. doi:10.1016/j.neurobiolaging.2015.11.010

Rantanen, T., Guralnik, J. M., Foley, D., Masaki, K., Leveille, S., Curb, J. D., \& White, L. (1999). Midlife hand grip strength as a predictor of old age disability. Journal of the American Medical Association, 281(6), 558560.

Reis, M. \& Arantes, P. (2011). Assessment of hand grip strength -- validity and reliability of the Saehan dynamometer. Fisioterapia e Pesquisa, 18(2), 176-181. Retrieved from http://search.ebscohost.com. proxy.li.suu.edu:2048/login.aspx?direct=true $\& d b=c i$ n20\&AN=104698057\&site=ehost-live

Roberts, H. C., Denison, H. J., Martin, H. J., Patel, H. P., Syddall, H., Cooper, C., \& Sayer, A. A. (2011). A review of the measurement of grip strength in clinical and epidemiological studies: Towards a standardised approach. Age and Ageing, 40(4), 423429. https://doi.org/10.1093/ageing/afr051

Ruprai, R., Tajpuriya, S., \& Mishra, N. (2016). Handgrip strength as determinant of upper body strength/physical fitness: A comparative study among individuals performing gymnastics (ring athletes) and gymnasium (powerlifters). International Journal of Medical Science and Public Health, 5(6), 1167. doi:10.5455/ijmsph.2016.09102015176

Sanborn, K., Boros, R., Hruby, J., Schilling, B., O’Bryant, H.S., Johnson, R.L., Hoke, T., Stone, M.E., Stone, M.H. (2000). Short-term performance effects of weight training with multiple sets not to failure vs a single set to failure in women. Journal of Strength and Conditioning Research, 14, 328-331.

Santanasto, A. J., Glynn, N. W., Lovato, L. C., Blair, S. N., Fielding, R. A., Gill, T. M., \& ... Newman, A. B. (2017). Effect of physical activity versus health education on physical function, grip strength and mobility. Journal of the American Geriatrics Society, 65(7), 1427-1433. doi:10.1111/jgs.14804

Schoffstall, J., Morrison, S.D., Kozlik, B., \& Boswell, B. (2010, February). Grip strength and powerlifting 
performance. In: Southeastern Chapter of the American College of Sports Medicine Regional Conference. Abstract retrieved from: https://www.researchgate.net/publication/28040887 8_Grip_Strength_and_Powerlifting_Performance

Shields, C.L., Whitney, F.E., \& Zomar, V.D. (1984). Exercise performance of professional football players. American Journal of Sports Medicine, 12, 455459.

Straub, W.F. (1979) Grip strength of college and professional football players. Ergonomics, 22(11), 1185-1194.

Suchomel, T., Nimphius, S., \& Stone, M. (2016). The Importance of Muscular Strength in Athletic Performance. Sports Medicine, 46(10), 1419-1449. Retrieved from http://search.ebscohost.com.proxy.li.suu.edu:2048/lo

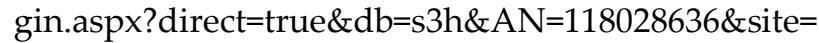
ehost-live

Wang, Y., Sindhu, B., Kapellusch, J., Bohannon, R. W., \& Xiaoyan, L. (2018). Hand-grip strength: Normative reference values and equations for individuals 18 to 85 years of age residing in the United States. Journal of Orthopedic E Sports Physical Therapy, 48(9), 685-693.

Wind, A. E., Takken, T., Helders, P. M., \& Engelbert, R. H. (2010). Is grip strength a predictor for total muscle strength in healthy children, adolescents, and young adults? European Journal of Pediatrics, 169(3), 281-287. doi:10.1007/s00431-009-1010-4

Young, V. L., Pin, P., Kraemer, B. A., Gould, R. B., Nemergut, L., \& Pellowski, M. (1989). Fluctuation in grip and pinch strength among normal subjects. The Journal of Hand Surgery, 14(1), 125-129. 